\title{
CORRIGENDUM
}

\section{Evidence for three genetic loci involved in both anorexia nervosa risk and variation of body mass index}

A Hinney, M Kesselmeier, S Jall, A-L Volckmar, M Föcker, J Antel, GCAN, WTCCC3, IM Heid, TW Winkler, GIANT, SFA Grant, EGG, Y Guo, AW Bergen, W Kaye, W Berrettini, H Hakonarson, Price Foundation Collaborative Group, Children's Hospital of Philadelphia/Price Foundation, B Herpertz-Dahlmann, M de Zwaan, W Herzog, S Ehrlich, S Zipfel, KM Egberts, R Adan, M Brandys, A van Elburg, V Boraska Perica, CS Franklin, MH Tschöp, E Zeggini, CM Bulik, D Collier, A Scherag, TD Müller and J Hebebrand

Molecular Psychiatry (2017) 22, 321-322; doi:10.1038/mp.2016.126; published online 26 July 2016

Correction to: Molecular Psychiatry advance online publication, 17 May 2016; doi:10.1038/mp.2016.71

Following the online publication of this paper, the first author noticed a mistake in Table 1. The corrected table accompanies this corrigendum.

The effect allele for anorexia nervosa (AN) was, despite several checks, wrongly assigned. The corrected data show that the alleles predisposing to AN also predispose to a lower body mass index (BMI). This finding is in accordance with a priori expectations based on previous research: (a) as of 1995, the genetic factors relevant in AN have been assumed to contribute to underweight. ${ }^{8,10,23-30}$ (b) More recently, LD-score regression analyses revealed a negative genetic correlation between AN and obesity. ${ }^{30}$

The incorrect direction of effect in Table 1 entails some incorrect wording in the main text. In detail: Mol Psychiatry advance online publication, May 17, 2016; doi:10.1038/mp.2016.71: Abstract, lines 8 and 9, the sentence should read: ' ...all AN susceptibility alleles were consistently associated with a lower BMI. ...'

On the fourth page, in the left hand column, lines 13 and 14, the sentence should read: '... Interestingly, for all SNPs, the AN risk alleles were consistently associated with a lower BMI (Table 1)...'

Table 1. Nine of the 1000 SNPs with the lowest $P$-values in a GWAS for AN risk $\left(\right.$ GCAN $\left.^{9}\right)$ are associated with decreased BMI $\left(\right.$ GIANT $^{11}$, with Bonferronicorrected $P<0.05$ significance; sorted according to the nominal $P$-values for BMI in all GIANT participants)

\begin{tabular}{|c|c|c|c|c|c|c|c|c|c|c|}
\hline $\begin{array}{l}\text { Chromosome/ } \\
\text { position/SNP } \\
\text { nearest gene(s) }\end{array}$ & Location & $\begin{array}{l}\text { Rank } \\
\text { in AN } \\
\text { GWAS }\end{array}$ & $\begin{array}{l}\text { AN reference } \\
\text { allele/ } \\
\text { frequency in } \\
\text { AN cases }\end{array}$ & $\begin{array}{l}\text { Odds ratio } \\
\text { (s.e.m.) for } \\
\text { AN reference } \\
\text { allele }\end{array}$ & $\begin{array}{c}\text { P-value for } \\
\text { AN risk }\end{array}$ & $\begin{array}{l}\text { BMI reference } \\
\text { allele/ } \\
\text { frequency in } \\
\text { GIANT }\end{array}$ & $\begin{array}{c}\beta \text { (s.e.m.) for } \\
\text { BMI } \\
\text { reference } \\
\text { allele }\end{array}$ & $\begin{array}{c}\text { Nominal P-value for } \\
\text { BMI: all } \\
\text { female/male }\end{array}$ & $\begin{array}{l}\text { Bonferroni } \\
\text { corrected } \\
\text { P-value }\end{array}$ & $\begin{array}{c}\text { Direction of } \\
\text { effect } \\
(+/-)^{\mathrm{b}}\end{array}$ \\
\hline $\begin{array}{l}10 / 126685663 \\
\text { rs1561589 } \\
\text { CTBP2 }\end{array}$ & Intron & 201 & G/0.67 & $1.14(0.04)$ & $7.74 \times 10^{-5}$ & G/0.66 & $\begin{array}{l}-0.0157 \\
(0.0033)\end{array}$ & $\begin{array}{c}2.47 \times 10^{-6} \\
3.45 \times 10^{-7} / 0.043\end{array}$ & 0.0025 & + \\
\hline $\begin{array}{l}10 / 126681170 \\
\text { rs } 12771627 \\
\text { CTBP2 }\end{array}$ & Intron & 190 & A/0.25 & $0.87(0.03)$ & $7.28 \times 10^{-5}$ & A/0.26 & $\begin{array}{c}0.0162 \\
(0.0035)\end{array}$ & $\begin{array}{c}4.25 \times 10^{-6} \\
5.8 \times 10^{-6} / 0.022\end{array}$ & 0.0043 & + \\
\hline $\begin{array}{l}10 / 126674064 \\
\text { rs11245456 } \\
\text { CTBP2 }\end{array}$ & Intron & 177 & $\mathrm{G} / 0.25$ & $0.87(0.03)$ & $6.79 \times 10^{-5}$ & $\mathrm{G} / 0.25$ & $\begin{array}{c}0.0171 \\
(0.0037)\end{array}$ & $\begin{array}{c}4.58 \times 10^{-6} \\
1.03 \times 10^{-5} / 0.009\end{array}$ & 0.0046 & + \\
\hline $\begin{array}{l}19 / 34978662 \\
\text { rs17513613 } \\
\text { CCNE1 }\end{array}$ & Distant $5^{\prime}$ & 409 & C/0.30 & $0.88(0.03)$ & 0.0002 & C/0.33 & $\begin{array}{c}0.015 \\
(0.0033)\end{array}$ & $\begin{array}{l}5.41 \times 10^{-6} \\
6.4 \times 10^{-3} / \\
1.24 \times 10^{-5}\end{array}$ & 0.0054 & + \\
\hline $\begin{array}{l}2 / 203492447 \\
\text { rs } 17406900 \\
\text { CARF }\end{array}$ & Intron & 709 & $\mathrm{G} / 0.52$ & $0.90(0.03)$ & 0.0003 & $\mathrm{G} / 0.51$ & $\begin{array}{c}0.0134 \\
(0.0031)\end{array}$ & $\begin{array}{l}1.08 \times 10^{-5} \\
1.8 \times 10^{-4} / \\
2.27 \times 10^{-3}\end{array}$ & 0.0108 & + \\
\hline $\begin{array}{l}\text { 2/203639257 } \\
\text { rs7593917 } \\
\text { NBEAL1 }\end{array}$ & Intron & 444 & G/0.54 & $0.89(0.03)$ & 0.0002 & $\mathrm{G} / 0.54$ & $\begin{array}{c}0.0131 \\
(0.0031)\end{array}$ & $\begin{array}{l}2.48 \times 10^{-5} \\
9.54 \times 10^{-5} / \\
9.39 \times 10^{-3}\end{array}$ & 0.0248 & + \\
\hline $\begin{array}{l}\text { 2/203582157 } \\
\text { rs11691351 } \\
\text { NBEAL1 }\end{array}$ & Distant $5^{\prime}$ & 412 & $\mathrm{G} / 0.54$ & $0.89(0.03)$ & 0.0002 & $\mathrm{G} / 0.54$ & $\begin{array}{c}0.0126 \\
(0.0031)\end{array}$ & $\begin{array}{l}3.57 \times 10^{-5} \\
1.98 \times 10^{-4} / \\
8.21 \times 10^{-3}\end{array}$ & 0.0357 & + \\
\hline $\begin{array}{l}19 / 34988693 \\
\text { rs8102137 } \\
\text { CCNE1 }\end{array}$ & Distant $5^{\prime}$ & 248 & C/0.30 & $0.88(0.03)$ & $9.45 \times 10^{-5}$ & C/0.33 & $\begin{array}{c}0.0169 \\
(0.0041)\end{array}$ & $\begin{array}{c}3.76 \times 10^{-5} \\
0.006 / 2.46 \times 10^{-4}\end{array}$ & 0.0376 & + \\
\hline $\begin{array}{l}2 / 203635796 \\
\text { rs7573079 } \\
\text { NBEAL1 }\end{array}$ & Intron & 401 & $\mathrm{~A} / 0.54$ & $0.89(0.03)$ & 0.0002 & $\mathrm{~A} / 0.54$ & $\begin{array}{c}0.0124 \\
(0.0031)\end{array}$ & $\begin{array}{c}4.61 \times 10^{-5} \\
2.80 \times 10^{-4} / 0.008\end{array}$ & 0.0461 & + \\
\hline $\begin{array}{l}\text { Abbreviations: } \\
\text { 2; GCAN, Genet } \\
\text { like 1, SNP, sin } \\
\text { decreased BMI }\end{array}$ & $\begin{array}{l}\text { anorexia r } \\
\text { husortium } \\
\text { AN risk a }\end{array}$ & $\begin{array}{l}\text { osa; B } \\
\text { AN; } \\
\text { olymo } \\
\text { dentic }\end{array}$ & $\begin{array}{l}\text { MMI, body ma } \\
\text { GIANT, Genet } \\
\text { rphism. }{ }^{\text {aPrir }} \\
\text { cal. }\end{array}$ & $\begin{array}{l}\text { index; CARF, } \\
\text { nvestigation } \\
\text { ry analysis, }\end{array}$ & $\begin{array}{l}\text { calcium-res } \\
\text { of ANthrok } \\
\text { sex-combin }\end{array}$ & correction & $\begin{array}{l}\text { NAS, genor } \\
1000 \text { tes }\end{array}$ & $\begin{array}{l}E 1 \text {, cyclin E1; CTBP } \\
\text { vide association st } \\
\text { irection of effect: }\end{array}$ & $\begin{array}{l}\text { s; NBEAL1, } \\
\text { the effec }\end{array}$ & $\begin{array}{l}\text { ding proteir } \\
\text { eurobeachin } \\
\text { isk allele fo }\end{array}$ \\
\hline
\end{tabular}


On the fourth page, in the left hand column, lines 28 and 29, the sentence should read: '... The direction of effect was opposite between AN risk and early onset extreme obesity in all five SNPs. ...'

On the fourth page, in the left hand column, lines 35 and 36, the sentence should read: '...For these SNPs the direction of effect was the same as the effect in GCAN.

On the fourth page, in the right hand column, lines 29 and 30, the sentence should read: '... However, for all available SNPs the direction of effect was opposite to that observed for obesity in the GIANT GWAMA...'
On the fourth page, in the right hand column, line 42 , the sentence should read: '... have indeed detected SNPs associated with both AN and lower BMI. ...'

On the fourth page, in the right hand column, lines 48-50, the sentences should read: '... It is also of interest that all AN risk alleles were consistently associated with lower BMI as ...'

On the sixth page, in the right hand column, lines $8-17$, the sentence should read: '...The finding that gene variants predisposing to a lower $\mathrm{BMI}$ are the same as those predisposing to $\mathrm{AN}$, is in accordance with a priori expectations based on previous research ${ }^{8,10,23-30} \ldots$...' 